\title{
ANÁLISE DOS EFEITOS DO DIÂMETRO DO DUTO DE UNDERFLOW E DA HASTE SÓLIDA INSERIDA EM UM HIDROCICLONE
}

\author{
J. L. MOGNON, J. M. SILVA, C. H. ATAÍDE \\ Universidade Federal de Uberlândia, Faculdade de Engenharia Química \\ E-mail para contato: chataide@ufu.br
}

\begin{abstract}
RESUMO - O núcleo gasoso ou o air core na parte central do hidrociclone é um fenômeno muitas vezes inevitável e ainda pouco compreendido, que altera o campo de escoamento rotacional da mistura no interior do separador. Diversos autores tem relacionado a ocorrência do air core ao aumento dos níveis de turbulência, efeito esse prejudicial ao processo de separação, principalmente a fração de partículas mais finas. Tendo em vista isso, esse trabalho visou analisar os efeitos da supressão do air core através da inserção de uma haste sólida ao longo de um hidrociclone modular de $30 \mathrm{~mm}$ de diâmetro característico. Além disso, foi possível descrever os efeitos individuais e combinados do diâmetro da haste inserida (4, 5 e $6 \mathrm{~mm}$ ) e o diâmetro do duto de underflow empregado $(6,7$ e $8 \mathrm{~mm})$. Os resultados obtidos mostraram uma redução das eficiências de coleta, aumento das divisões de fluxo e incremento na capacidade de processamento para a operação com as hastes, o que de forma geral não justifica os custos de sua instalação nos equipamentos atuais.
\end{abstract}

\section{INTRODUÇÃO}

Hidrociclones, também denominados ciclones hidráulicos, consistem em dispositivos amplamente utilizados na indústria, com destaque para o setor da mineração, como sistemas extremamente versáteis de separação (sólido-líquido e líquido-líquido) a mais de 100 anos (Wills e Napier-Munn, 2006). A expansão da aplicação industrial de tal equipamento começou por volta do final da década de 40, inicialmente nas indústrias de extração e processamento mineral. Desde então, sua utilização foi difundida, sendo atualmente um equipamento de separação bastante usado nas indústrias química, petroquímica, metalúrgica, dentre outras. $\mathrm{O}$ fato da ampla presença desta tecnologia no setor industrial deve-se fundamentalmente, ao seu tamanho reduzido quando comparado com outros tipos de separadores, ausência de partes móveis, baixo custo de instalação e operação e principalmente a sua flexibilidade operacional.

\subsection{Escoamento no interior do hidrociclone}

A mistura de líquidos ou sólido-líquido é alimentada tangencialmente na câmara cilíndrica gerando um escoamento helicoidal que forma um campo centrifugo dirigindo as partículas (ou 


\section{9 a 22 de outubro de 2014 \\ Florianópolis/SC}

líquido) mais densas para a parede interna do equipamento. Na região cônica, o fluido sofre uma restrição de escoamento e parte do fluxo esse é direcionado para a saída superior (overflow) onde se concentram as partículas de menor diâmetro ou menor densidade, enquanto que o fluxo junto a parede é coletado pela saída inferior (underflow).

As altas velocidades tangenciais, associadas ao movimento helicoidal do fluido ao longo do hidrociclone criam um gradiente de pressão em direção radial do equipamento, resultando assim em um núcleo de baixa pressão alinhado ao eixo central deste. Nessa região, devido a própria dinâmica de separação entre componentes de diferentes densidades e ao fato de apresentar pressões inferiores à atmosférica, forma-se um núcleo gasoso comumente denominado air core. Segundo Neesse e Dueck (2007) o ar presente nesse núcleo provém tanto de bolhas presentes na corrente de alimentação quanto da sucção de gás pelo duto de underflow.

\subsection{Revisão Bibliográfica}

A inserção de hastes no interior de hidrociclones tem sido estudada e descrita por diversos autores, dentre eles pode-se destacar os trabalhos de Sripriya et al (2012) e Chu et al (2000). Esses autores relataram aumentos significativos nas eficiências de separação e redução do diâmetro de corte através da inserção de uma haste metálica sólida ao longo eixo central do corpo do hidrociclone, com o objetivo principal de suprimir a formação do núcleo de ar denominado air core. Segundo os autores citados a justificativa para tal melhoria de desempenho se fundamenta na estabilização das linhas de corrente no interior do hidrociclone, tendo-se em vista que a presença do air core tem caráter altamente turbulento e instável. Os autores observaram também uma redução nas velocidades radial e axial juntamente com um aumento da velocidade tangencial na região cônica do hidrociclone. Esses efeitos podem explicar uma maior recuperação das partículas (principalmente a fração mais fina). De outro modo, Lee e Willians (1993) realizaram um trabalho pioneiro no que se refere a inserção de hastes e supressão do air core em hidrociclones. Em seus resultados constatou-se que essa modificação estrutural não apresentou benefícios no que tange eficiência de separação e razão de líquido, o que vai em sentido contrário aos autores mais recentes, abrindo espaço para futuras discussões e outras pesquisas no assunto.

\subsection{Objetivo}

O objetivo do presente estudo é analisar o efeito da combinação de diferentes diâmetros de underflow e diâmetros de haste sólida, inseridas no interior de um hidrociclone, com o propósito de suprimir o efeito do air core.

\section{MATERIAIS E MÉTODOS}

\subsection{Unidade Experimental}

Um esquema da unidade experimental utilizada neste trabalho, montada no Laboratório da Unidade Avançada de Pesquisa da Faculdade de Engenharia Química da Universidade Federal de Uberlândia, encontra-se na Figura 1. 


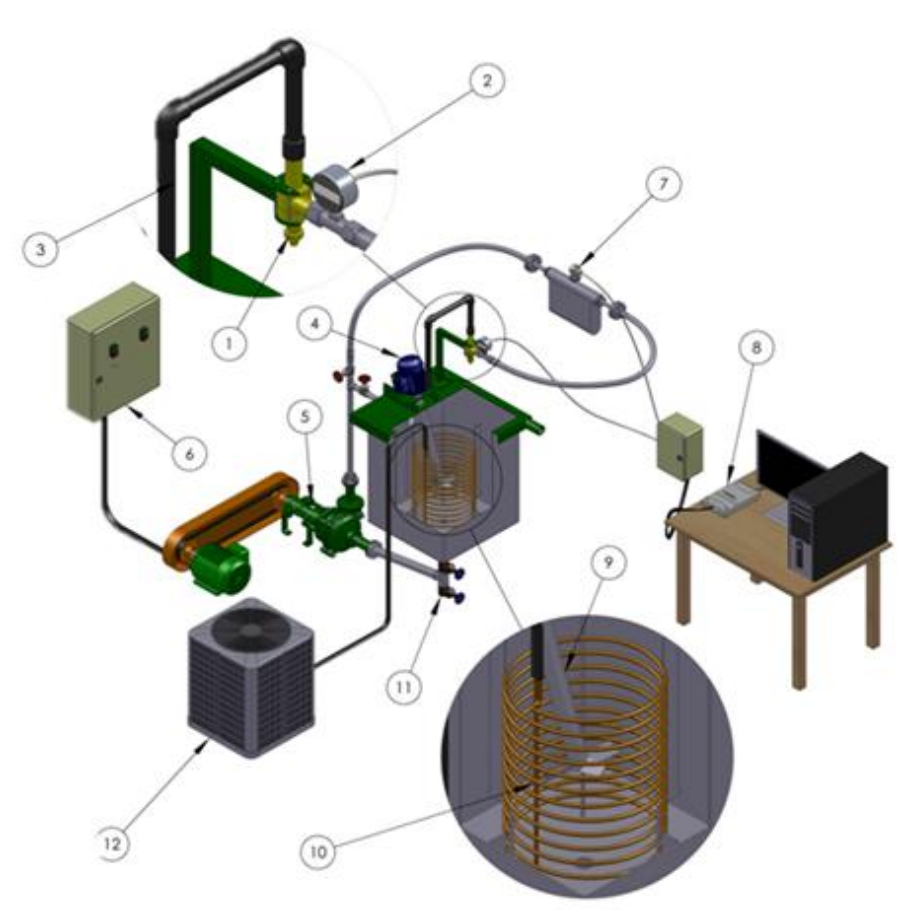

1. Hidrociclone;

2. Manômetro digital;

3. Tubulação para overflow;

4. Motor do agitador;

5. Bomba centrifuga de $10 \mathrm{cv}$;

6. Quadro elétrico;

7. Medidor de vazão (Coriólis);

8. Placa de aquisição de dados;

9. Agitador;

10. Serpentina para resfriamento;

11. Válvulas do tipo gaveta;

12. Unidade de refrigeração.

Figura 1 - Unidade de hidrociclonagem.

\subsection{Hidrociclone}

O hidrociclone utilizado nos experimentos consiste em uma geometria baseada no hidrociclone fabricado pela empresa Derrick (atualmente utilizado como dessiltador no controle de sólidos da perfuração de poços de petróleo e gás), fabricado em poliuretano em menor escala (1:3) com relação ao comercial. As principais dimensões do hidrociclone utilizado são apresentadas na Figura 2.

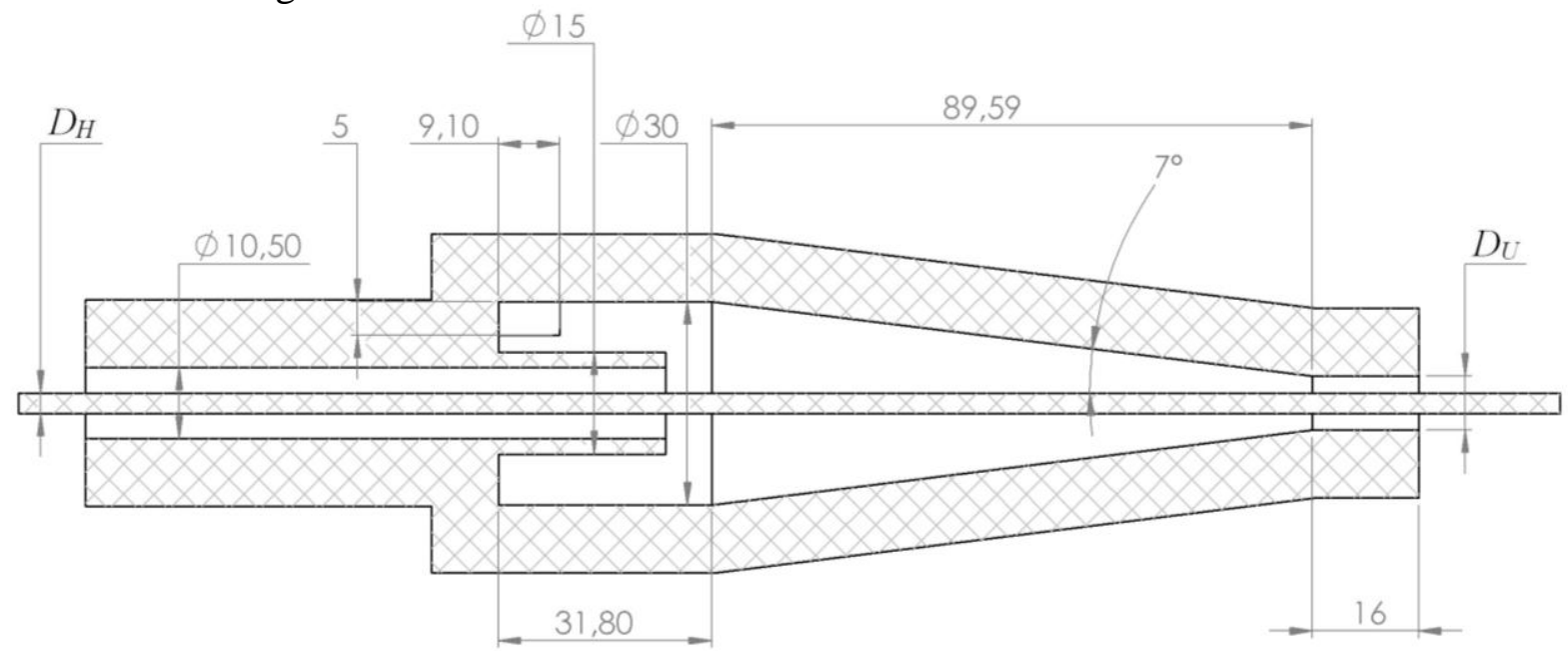

Figura 2 - Dimensões do hidrociclone (dimensões em milímetros). 


\subsection{Material Particulado}

Para os ensaios utilizou-se água industrial contendo uma quantidade de $0,5 \%$ (em volume) de rocha fosfática fina. A granulometria do material em questão foi obtida através do método de difração de raio laser (Mastersizer 2000) e é apresentada na Figura 3.

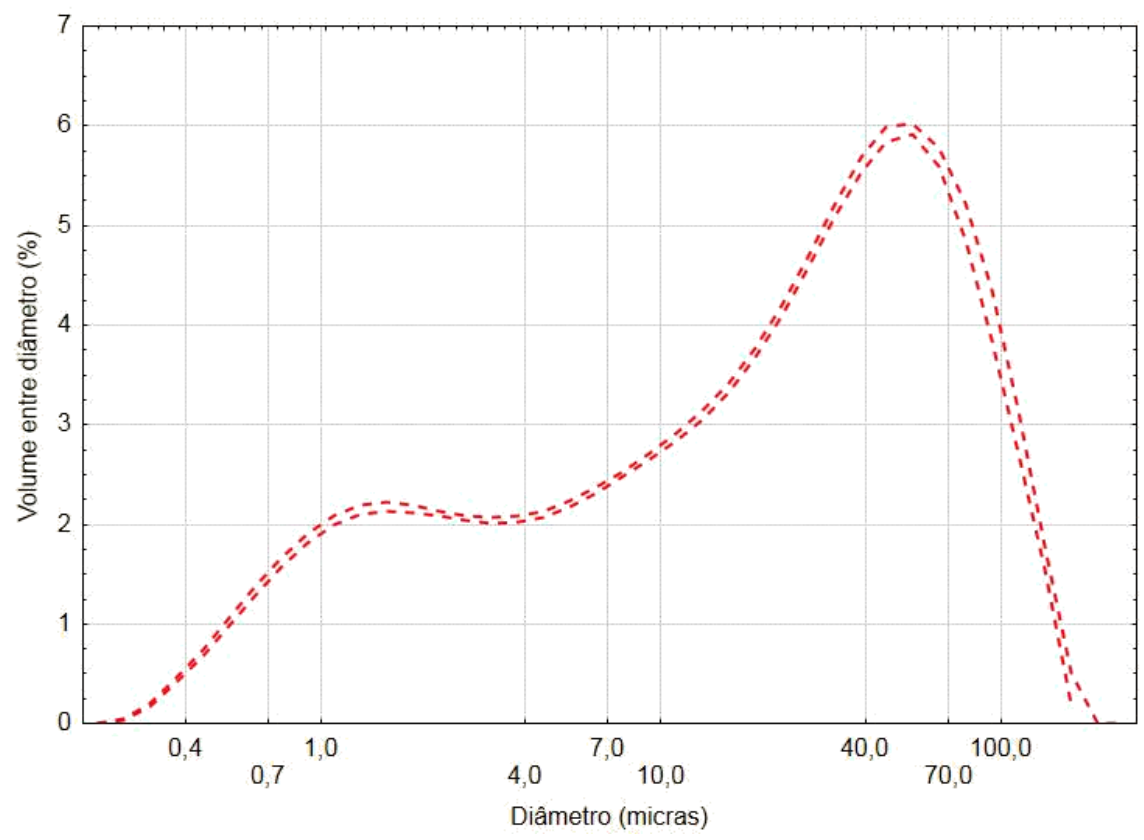

Figura 3 - Granulometria do material particulado.

\subsection{Planejamento experimental}

O planejamento experimental utilizado compreendeu três níveis de diâmetro de underflow $(6,7$ e $8 \mathrm{~mm})$ e diâmetro de haste $(4,5$ e $6 \mathrm{~mm})$ instalados no hidrociclone modular. Somado-se a isso foram realizados ensaios sem a presença da haste sólida.

Deve-se salientar que a geometria com $D_{U}=6 \mathrm{~mm}$ e $D_{H}=4 \mathrm{~mm}$ não pode ser analisada pela ocorrência de instabilidade operacional (vibração e rompimento da haste). Além disso, a geometria onde $D_{U}=6 \mathrm{~mm}$ e $D_{H}=6 \mathrm{~mm}$ não foi testada pela completa obstrução do duto de underflow e impossibilidade de análise do equipamento. Com isso o planejamento realizado contemplou um total de 10 experimentos independentes.

\subsection{Metodologia experimental} seguir:

A metodologia utilizada na preparação dos ensaios e análise dos resultados é descrita a 
- Preparação da suspensão contendo 160 litros de água e cerca de $5 \mathrm{~kg}$ de rocha fosfática. A mistura foi mantida sobre constante agitação de modo a evitar a sedimentação do material particulado.

- Ajuste da vazão de alimentação em 360 g/s, através de sistema de controle proporcional acoplado ao inversor de frequência da bomba.

- Medição das vazões mássicas da lama nas correntes de underflow e overflow por gravimetria.

- Coleta em triplicata de amostras das correntes de underflow e overflow, seguida por secagem em estufa a $107^{\circ} \mathrm{C}$ por 24 horas.

A temperatura dos experimentos foi continuamente verificada, situando-se na faixa de 23 à $25^{\circ} \mathrm{C}$.

\section{RESULTADOS E DISCUSSÃO}

\subsection{Parâmetros de análise}

Os parâmetros selecionados para determinação das condições ótimas de operação são apresentados a seguir:

Eficiência total de separação $\left(E_{t}\right)$ : corresponde a porcentagem de sólidos coletados no duto de underflow em relação a quantidade alimentada, pode ser expressa pela Equação 1.

$$
E_{t}(\%)=100 \frac{C_{u} W_{u}}{C_{a} W_{a}}
$$

Razão de líquido $\left(R_{L}\right)$ : corresponde a porcentagem de líquido coletado no duto de underflow com relação ao total alimentado, pode ser expressa pela Equação 2.

$$
R_{L}(\%)=100 \frac{W_{u}\left(1-C_{u}\right)}{W_{a}\left(1-C_{a}\right)}
$$

Número de Euler $(E u)$ : corresponde a quantidade de energia necessária para a manutenção de determinada vazão através do equipamento (Equação 3).

$$
E u=\frac{\Delta P}{\left(\rho_{\mathrm{H}_{2}} \mathrm{O}_{c}\right) / 2}
$$

\subsection{Dados obtidos nos experimentos}

Na Tabela 1 são apresentados os dados obtidos nos experimentos realizados segundo o planejamento proposto.

Pode-se observar uma redução expressiva no número de Euler para os ensaios as geometrias que apresentam a inserção da haste. Isso pode ser explicado pela redução dos níveis 
de turbulência obtida através da supressão do air core e pelos efeitos de parede presentes com a inserção das hastes.

Tabela 1 - Dados experimentais obtidos obtidos.

\begin{tabular}{|ccccc|}
\hline$D_{H}(\mathrm{~mm})$ & $D_{U}(\mathrm{~mm})$ & $R_{L}(\%)$ & $E_{t}(\%)$ & $E u$ \\
\hline 6 & 8 & 35,91 & 86,93 & 825,81 \\
\hline 6 & 7 & 14,85 & 79,41 & 885,37 \\
\hline 5 & 8 & 34,87 & 86,85 & 812,27 \\
\hline 5 & 7 & 25,38 & 82,77 & 839,35 \\
\hline 5 & 6 & 16,84 & 81,21 & 861,01 \\
\hline 4 & 8 & 33,93 & 86,77 & 866,42 \\
\hline 4 & 7 & 22,00 & 83,44 & 888,08 \\
\hline 0 & 8 & 34,22 & 87,42 & 871,84 \\
\hline 0 & 7 & 22,65 & 84,17 & 907,03 \\
\hline 0 & 6 & 18,04 & 83,30 & 920,57 \\
\hline
\end{tabular}

\subsection{Eficiência total de separação}

A Figura 4 ilustra os valores de eficiência total obtidos para as geometrias analisadas segundo a Tabela 1. Pode-se observar que há um aumento da eficiência de separação com o aumento do diâmetro de underflow, esse efeito já era esperado pelo fato do aumento da divisão de fluxo e consequente carregamento de maior quantidade de material particulado pela corrente de underflow.

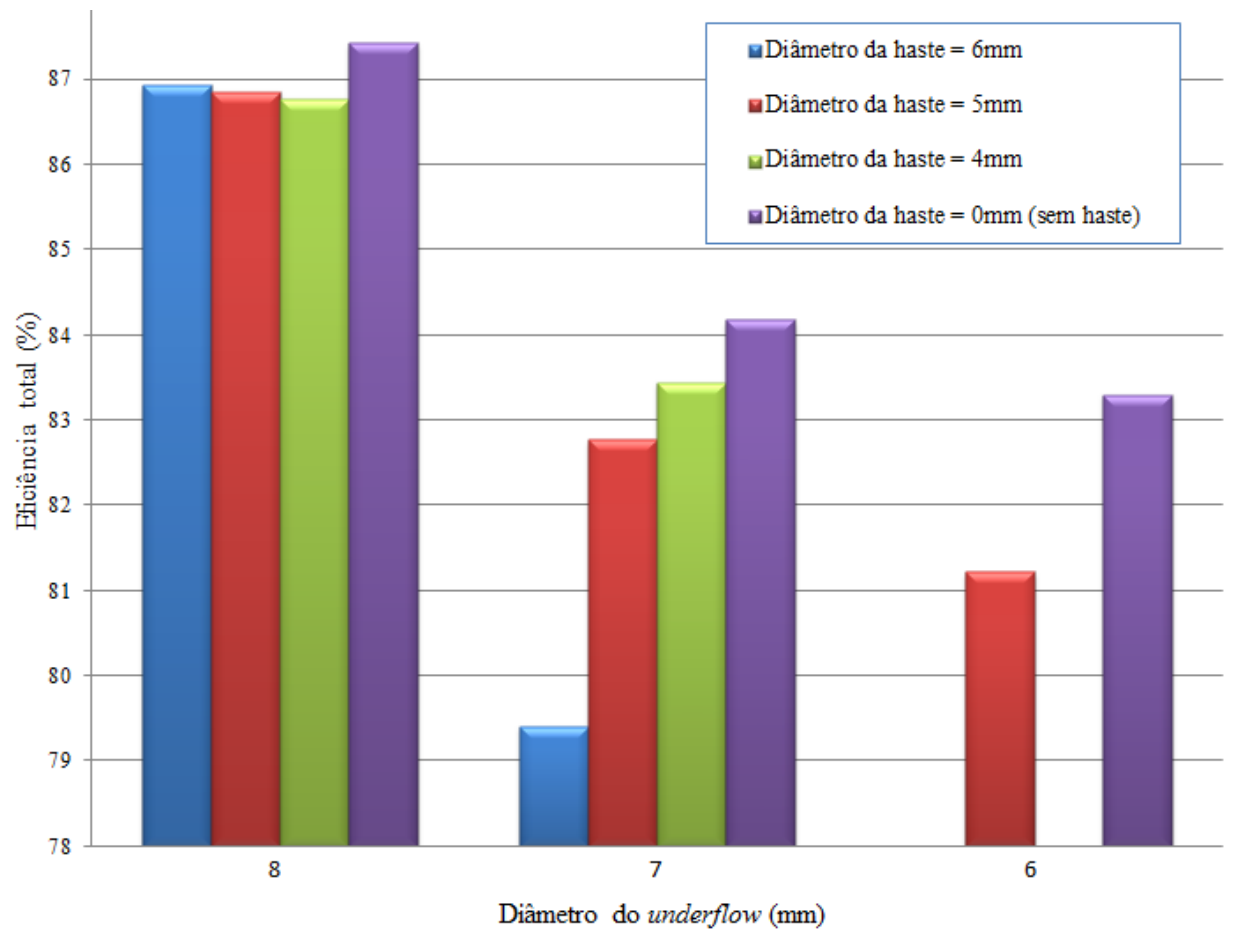

Figura 4 - Eficiência total das geometrias testadas. 
Com relação a presença das hastes, a Figura 4 mostra que houve uma redução de eficiência para todas as configurações com haste. Essa redução foi mais expressiva quanto menor o diâmetro do duto de underflow. Esse efeito pode ser explicado pela redução na área total da seção de underflow que ocorre pelo aumento do diâmetro da haste ou pela própria redução do diâmetro do duto inferior.

\subsection{Razão de líquido}

A Figura 5 apresenta os valores de razão de líquido para todas as combinações de geometria testadas. Pode-se observar que para grande parte das configurações houve um aumento da razão de líquido com a utilização de hastes mais espessas. Esse efeito é diferente do esperado, já que há uma redução na área da seção de underflow, o que deveria acarretar em um aumento da divisão de fluxo.

Uma possível explicação para esse fenômeno seria o controle das estruturas de turbulência e estabilização das linhas de fluxo no interior do equipamento, o que reduziria as perdas de energia cinética do fluido na direção descendente, aumentando assim a quantidade de líquido coletado do underflow.

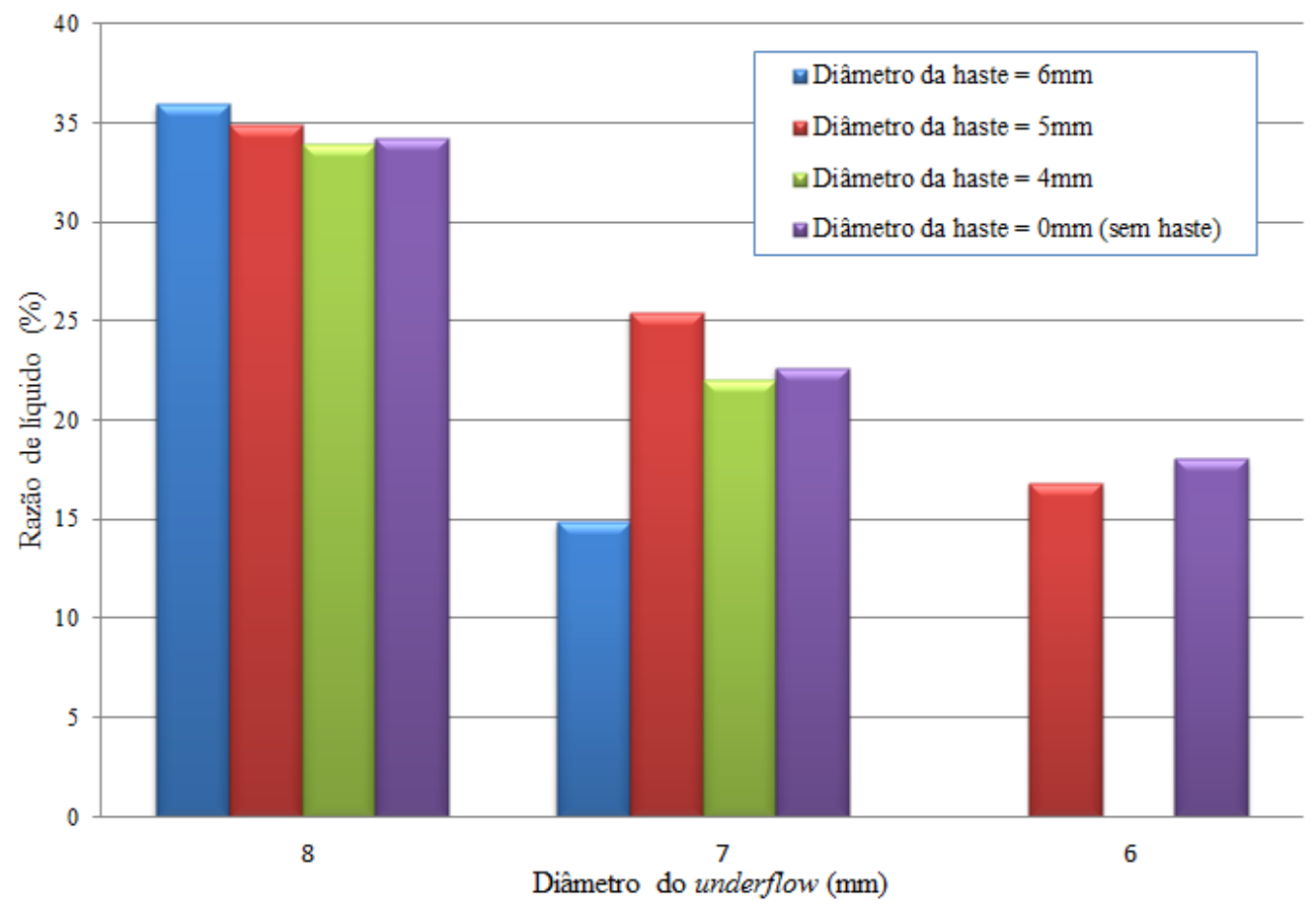

Figura 5 - Razão de líquido das geometrias testadas.

\section{CONCLUSÕES}

Os resultados obtidos segundo o planejamento experimental proposto mostraram que a inserção de hastes sólidas não apresentam ganhos concretos na eficiência de operação dos 
hidrociclones. Em todos os casos estudados, essas modificações apresentaram redução da eficiência total de coleta, e em diversas situações um aumento da razão de líquido do equipamento. Por outro lado, a capacidade de operação aumentou com a presença das hastes, o que de modo geral não compensa os custos de instalação e manutenção da modificação em questão.

\section{NOMENCLATURA}

$C_{a}$ : concentração mássica de alimentação [-]

$C_{u}$ : concentração mássica do underflow $[-]$

$\Delta P:$ queda de pressão no hidrociclone [psi]

$D_{H}$ : diâmetro das hastes [mm]

$D_{U}$ : diâmetro do duto de underflow [mm]

$E_{t}$ : eficiência total [\%]

$E u$ : número de Euler [-]

$R_{L}$ : razão de líquido [\%]

$v_{c}$ : velocidade na câmara cilíndrica $[\mathrm{m} / \mathrm{s}]$

$W_{a}$ : vazão mássica da alimentação $[\mathrm{g} / \mathrm{s}]$

$W_{u}$ : vazão mássica do underflow $[\mathrm{g} / \mathrm{s}]$

\section{REFERÊNCIAS BIBLIOGRÁFICAS}

CHU, L., CHEN, W., LEE, X. Effect of structural modification on hydro-cyclone performance. Separation and Purification Technology., Vol. 21, p. 71-86, 2000.

LEE, M. S., WILLIANS, R.A. Performance characteristics within a modified hydrocyclone. Minerals Engineering, Vol. 6, No 7, p. 743-751, 1993.

NEESSE, T., DUECK, J. Air core formation in the hydrocyclone. Journal of Minerals Engineering, Vol. 20, p. 349-354, 2007.

SRIPRIYA, R., SURESH, N., CHANDRA,S., BHATTACHARJEE, D. The effect of diameter and height of the inserted solid rod in a dense medium cyclone to suppress air core. Minerals Engineering, Vol. 42, p. 1-8, 2013.

WILlS, B. A., NAPIER-MUNN, T. Wills' Mineral Processing Technology, Seventh Ed. Butterworth-Heinemann, Boston, 2006.

\section{AGRADECIMENTOS}

Agradecemos a Fundação de Amparo à Pesquisa do Estado de Minas Gerais (FAPEMIG) pelos recursos concedidos no Projeto de Participação Coletiva em Eventos Técnicos-Científicos (PCE-00082-14). 